\title{
Study on Warrant Application under GARCH-Mote Carlo Algorithm
}

\author{
Ruiyi LIU, Zengwen LIU * and Fengxia WANG \\ Shandong University, Jinan, 250100, China
}

\begin{abstract}
In this paper, the GARCH $(1,1)$ model is used for data modeling and forecasting. The GARCH $(1,1)$ model is combined with the Mote Carlo (MC) algorithm to study European option pricing. The differences between the model data and the observed data are calculated by the GARCH-MC model. The GARCH-MC model is adjusted according to the differences to forecast the option prices. The GARCH-MC model is tested by the SGCOC and the SIC warrants. It is concluded that the variation trend of the model data and the observe data are coincident. The GARCH-MC model can be used to forecast accurately the option prices after adjusting.
\end{abstract}

KEYWORD: GARCH Model; Monte Carlo Algorithm; European Option; Option Pricing

\section{INTRODUCTION}

After the 2008 financial crisis, the risk of fluctuations in the financial assets had gradually become a hot topic. In a market economy, these fluctuations are not only to bring a profit but also become a risk to securities and options. Currently, the study of time series of stock market volatility has become an integral part of the financial study. Therefore, it is of very important to study the timeseries analysis of financial assets.

In recent years, a large number of domestic and foreign scholars promote the progress of financial time series analysis. Engle (1982) proposed a creative autoregressive heteroscedasticity model (ARCH model) to forecast the correlation of error conditional variance [1]. The ARCH model was developed by Bollerslev ( 1986 ) to build a generalized autoregressive heteroskedasticity model (GARCH model) [2]. Taufiq (1995) used GARCH model to study the volatility characteristics of 19201930 five European countries stock returns and found that financial assets would continue to fluctuate with the interference of external factors [3]. Li Cong (2006) used the GARCH model to calculate the Shenzhen Composite Index's value depending on the different distribution function, and found that GARCH model had some limitations [4]. Liu and Hung (2010) forecasted S\&P100 Index volatility equation in the case of asymmetric information, and found that asymmetric GARCH model was more accurate in predicting volatility [5].
Although GARCH model has been relatively mature in the study of volatility, but the existing method isn't simple enough in solving the European option pricing problem. In this article, GARCH model and Monte Carlo algorithm (GARCH-MC) will be combined to calculate the future value of the stock by means of simulating the underlying price's changing track. The warrants will be used to conduct empirical test.

\section{MODELING}

\subsection{Theoretical basis}

The chromatography column was successively filled by cotton, $5.5 \mathrm{~g}$ silica gel with column chromatography reagent grade (100-200 mesh, activation for 5 hours at $120^{\circ} \mathrm{C}$ ), and $1.2 \mathrm{~g}$ anhydrous sodium sulfate (AR, activation for $12 \mathrm{~h}$ at $30^{\circ} \mathrm{C}$ ) placed on the top. Each sample accurately weighed was fully dissolved by $15 \mathrm{~mL}$ configured mixed solution of dichloromethane and n-hexane (volume ratio is 2:1). Rinse the filled column by $10 \mathrm{~mL}$ hexane, when anhydrous sodium sulfate just exposed, immediately pouring the dissolved oil sample prepared into the column and collecting the chromatography liquid for testing.

Monte Carlo method is a computing method based on probability theory and statistical methods. In this method, the issue to be solved is linked with a probability model. The approximate solution will be found out by means of simulating in computer. 
Monte Carlo method is based on risk-neutral pricing. European derivative securities are studied in this paper, and such derivative securities holders cannot make any decisions within the validity period. Assuming that at the moment $T$, the earnings of derivative securities is $f_{T}$. Then in the neutral world, the value of the time 0 follows the following equation.

$$
f=e^{r T} \hat{E}\left[f_{T}\right]
$$

Where $r$ is risk-free rate.

The path of the underlying variables in a riskneutral world can be simulated, if derivative securities only depend on a random variable, and this variable is not the interest rate. Then, thereturnis calculated in the path back to time 0 . The final value of the derivative securities is obtained, which may be seen as the random sample of the all final value collection.

After calculating the final value of large number of samples, $\hat{E}\left[f_{T}\right]$ is estimated by the arithmetic mean of final values. By discounting the risk-free interest rate in $\hat{E}\left[f_{T}\right]$, the present value of the estimated value of the derivative securities can be calculated.

When researching fluctuations of financial assets, the volatility is usually used as a measure of the size of the index fluctuations. The underlying price series $\left\{S_{t}\right\} \quad(t=0,1, \mathrm{~L} \mathrm{~L})$ is converted into the underlying the return sequence $\left\{u_{t}\right\} \quad(i=1,2, \mathrm{~L} \mathrm{~L})$ to consider. $\left\{u_{t}\right\}$ is defined as the following equation.

$$
u_{t}=\ln \left(\frac{S_{t-1}}{S_{t}}\right)
$$

In the risk-neutral world, suppose that the return sequence of stocks follows the following equation.

$$
u_{t}=\ln \left(\frac{S_{t}}{S_{t 1}}\right)=+_{t}
$$

Where $\mu$ is mean, $\varepsilon_{t}$ is random variable, also ${ }_{t} t_{t 1} \sim N\left(0, \begin{array}{ll}2 \\ t\end{array}\right), \Psi_{t-1}$ means all the information sets before the moment $t-1$.

$\left\{\sigma_{t}\right\} \quad(t=l+1, l+2, \mathrm{~L})$ can be got by $\left\{u_{t}\right\}$ ( $i=1,2, \mathrm{~L} \mathrm{~L} \quad$ ). $\left\{\sigma_{t}\right\} \quad$ is defined as following equation.

$$
\sigma_{t+1}=\sqrt{\frac{1}{l-1} \sum_{j=t-l+1}^{t}\left(u_{j}-\bar{u}\right)^{2}}
$$

Where, $l$ is window length.

Then, ${ }_{t}^{2}$ follows $\operatorname{GARCH}(1,1) \operatorname{model}[6]$.

$$
\begin{aligned}
& { }_{t}^{2}=+\left(u_{t 1}\right)+{ }_{t 1}^{2} \\
& \omega=\gamma V_{L}
\end{aligned}
$$

Where $\alpha, \beta, \gamma$ are corresponding parameters, $V_{L}$ is long-term average variance, and $\alpha+\beta+\gamma=1$.

\section{Parameter estimation [7]:}

Assuming $v_{i}=\sigma_{i}^{2}$ is the estimate of variance at the moment $i$ under the condition of a given variance, and the probability distribution of $\varepsilon_{i}$ is normal distribution, it follows the following equations.

$$
\begin{gathered}
u_{i}=\ln \left(\frac{S_{i}}{S_{i-1}}\right)=\mu+\varepsilon_{i} \\
\sigma_{i}^{2}=\frac{1}{i} \sum_{j=1}^{i}\left(u_{j-1}-\mu\right)^{2}
\end{gathered}
$$

Then the likelihood function is $\prod_{i=1}^{m}\left[\frac{1}{\sqrt{2 \pi v_{i}}} \exp \left(\frac{-\left(u_{i}-\mu\right)^{2}}{2 v_{i}}\right)\right]$.

After taking the logarithm, it is $\sum_{i=1}^{m}\left[-\ln \left(v_{i}\right)-\frac{\left(u_{i}-\mu\right)^{2}}{v_{i}}\right]$.

The likelihood function is calculated by using the search algorithm for each group $\alpha 、 \beta$ 、 $\omega$ to calculate, and the optimal solution is obtained (The optimal parameters can be carried out by using the SPSS 20.0).

After getting the optimal parameters, $\operatorname{GARCH}(1,1)$ model is used to forecast volatility in the future. It follows the following equation.

$$
\begin{gathered}
E\left[\sigma_{n+t}^{2}-V_{L}\right]=(\alpha+\beta)^{t}\left(\sigma_{n}^{2}-V_{L}\right) \text { or } \\
E\left[\sigma_{n+t}^{2}\right]=V_{L}+(\alpha+\beta)^{t}\left(\sigma_{n}^{2}-V_{L}\right)
\end{gathered}
$$

\subsection{GARCH-MC option pricing steps}

Step1: Creating a standard normal random matrix with $j$ lines and $T$ columns, where $T$ means the number of days to the deadline (Time to deadline excluding weekends and holidays), $j$ means the number of the simulation path, $n(j, T)$ means an element of the standard normal random matrix. And $n(j, i) \sim N(0,1) i=1,2,3, \mathrm{~L}, T$

Step2: The volatility at the moment $T$ is calculated by using the $\alpha$ 、 、 $\omega$ coming from $\operatorname{GARCH}(1,1)$ estimation. And then, the estimation of volatility is got as $\hat{\sigma}_{t+1}, \hat{\sigma}_{t+2}, \hat{\sigma}_{t+3}, \mathrm{~L}, \hat{\sigma}_{t+T}$.

Then the estimation of $\hat{u}_{t+i}$ is obtained as the following equation. 
$\hat{u}_{t+i}=\hat{\sigma}_{t+i} n(j, T)+\mu$

Step3: Under the known condition of $S_{t}$, the stock price at time $T$ follows the following equation.

$$
\hat{S}_{t+T}=S_{t} \exp \left\{\sum_{l=1}^{T} \hat{u}_{i+l}\right\}
$$

Step4: Then, the European option prices follow the following equations.

$$
\begin{aligned}
& c=e^{-r_{0} T} \max \left\{\hat{S}_{t+T}-K, 0\right\} \\
& p=e^{-r_{0} T} \max \left\{K-\hat{S}_{t+T}, 0\right\}
\end{aligned}
$$

Step5: After averaging the simulation $j$ times, the option price is carried out.

\section{EMPIRICAL TEST}

The fitting degree of the option price estimated by the model and the real market price is the most concerning factor in option pricing. Three indicators are used to measure the fitting degree of the GARCH-MC model. They are the Mean Absolute Error (MAE), the Mean Relative Percentage Error (MRPE) and the Mean Absolute Percentage Error (MAPE). Relative to MAE, MRPE is a percentage of the value, which can better characterize the relationship between the error value and the option price. Compared to MRPE, MAPE is an absolute value data, which is a positive number and more convenient to analyze.

$$
\begin{aligned}
& M A E=\frac{1}{N} \sum\left|P_{\text {model }}-P_{o b s}\right| \\
& M R P E=\frac{1}{N} \sum\left(\frac{P_{\text {model }}}{P_{o b s}}-1\right) \\
& M A P E=\frac{1}{N} \sum\left|\frac{P_{\text {model }}}{P_{o b s}}-1\right|
\end{aligned}
$$

Where, $N$ is the data size, $P_{\text {model }}$ is the model data, $P_{o b s}$ is the observed data.

The observed data is from the State Grid Corporation Of China (SGCOC) shares and warrant data, the Sinochem International Corporation (SIC) shares and warrant data.

\subsection{SGCOC pricing results}

The comparison of the model data and the observed data of SGCOC warrant is shown in Fig. 1.

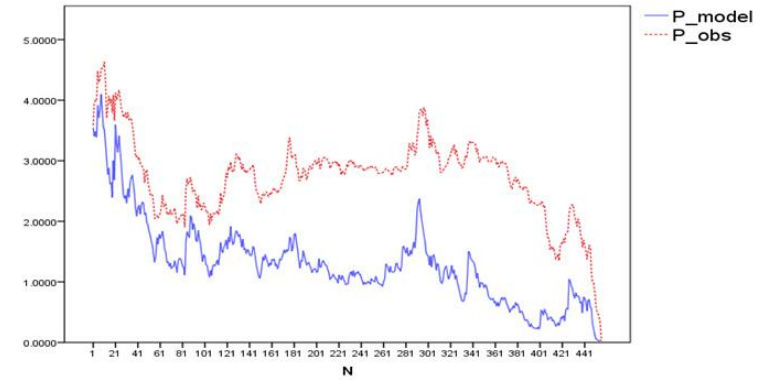

Fig.1 The comparison of the model data and the observed data of SGCOC warrant

The MAE, MRPE and MAPE between model data and observed data are shown in table 1 during 457 market days.

Table 1: Three indicators of SGCOC Pricing

\begin{tabular}{|c|c|c|c|}
\hline & MAE & MRPE & MAPE \\
\hline Mean & 1.4176 & .5345 & 5357 \\
\hline $\mathrm{N}$ & 457 & 457 & 457 \\
\hline Standard Deviation & .52945 & .19134 & .18794 \\
\hline
\end{tabular}

It can be seen from Fig. 1 that there are some differences between model data and observed data. It also can be seen from Table 1 that the values of MAE, MRPE and MAPE are larger. But the variation trend of the model data and the observed data is coincident. It shows that the model data can't be used directly to forecast the options price. The model should be adjusted according to the differences between model data and observed data.

$$
P \_ \text {adjusted }=P \_ \text {model }+D_{\text {price }}
$$

Where, $D_{\text {price }}$ is the mean value of the differences between model data and observed data.

The comparison of the adjusted model data and the observed data of SGCOC warrant is shown in Fig.2. Three indicators of SGCOC Pricing afer adjusting are shown in Table 2.

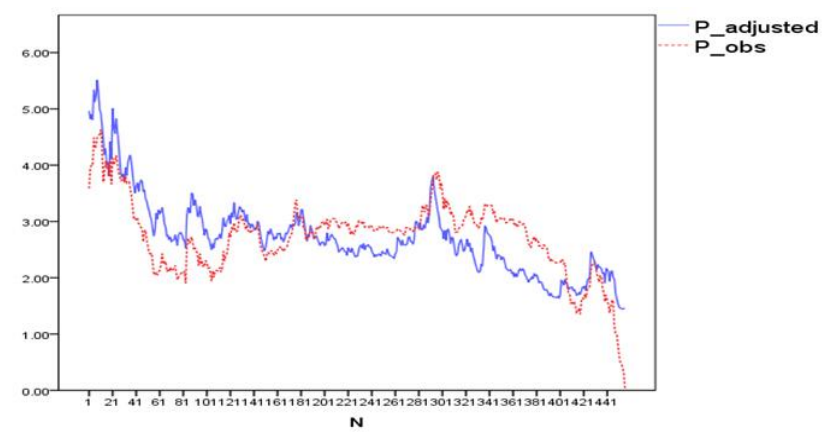

Fig. 2 The comparison of the adjusted model data and the observed data (SGCOC)

Table 2:Three indicators of SGCOC Pricing afer adjusting

\begin{tabular}{|c|c|c|c|}
\hline & MAE & MRPE & MAPE \\
\hline Mean & 0 & 0 & .1056 \\
\hline $\mathrm{N}$ & 457 & 457 & 457 \\
\hline Standard Deviation & .52945 & .19134 & .18794 \\
\hline
\end{tabular}


As can be seen from the Fig. 2, the differences are reduced after adjusting. It also can be seen from Table 2 that the values of MAE, MRPE and MAPE are also reduced dramatically after adjusting. It shows that the model data after adjusting is fit for SGCOC warrant pricing and GARCH-MC model after adjusting can be used to forecast the options price.

\subsection{SIC pricing results}

The comparison of model data and observed data of SIC warrant is shown in Fig.3.

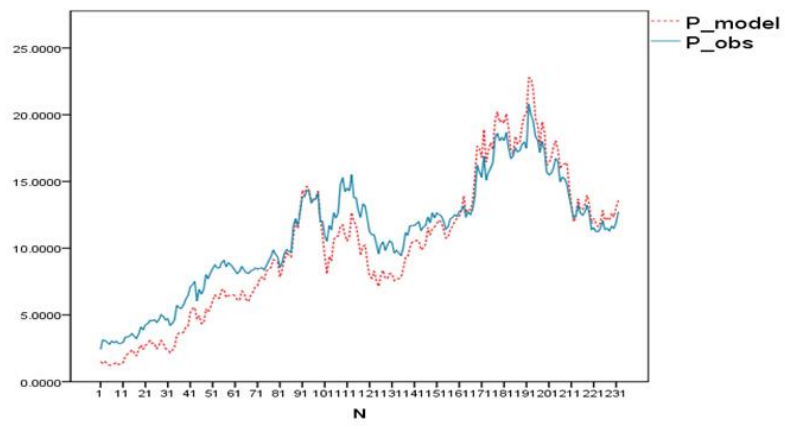

Fig.3The comparison of the model data and the observed data of SIC warrant

The MAE, MRPE and MAPE between model data and observed data are shown in table 2 during 232 market days.

Table3: Three indicators of SIC Pricing

\begin{tabular}{|c|c|c|c|}
\hline & MAE & MRPE & MAPE \\
\hline Mean & 1.1561 & .0144 & .1125 \\
\hline $\mathrm{N}$ & 232 & 232 & 232 \\
\hline Standard Deviation & 1.01266 & .14471 & .09187 \\
\hline
\end{tabular}

As can be seen from the Fig. 3, the theoretical price and the market price are pretty close under the GARCH-MC model estimating. It also can be seen from Table 3 that the PRE and APRE are small. It shows that the model data is fit for SCI warrant pricing and GARCH-MC model can be used directly to forecast the options price. But adjusting according to Eq. 17, the accuracy of the model is higher.

The comparison of the adjusted model data and the observed data of SIC warrant is shown in Fig.4. Three indicators of SIC Pricing afer adjusting are shown in Table 4.

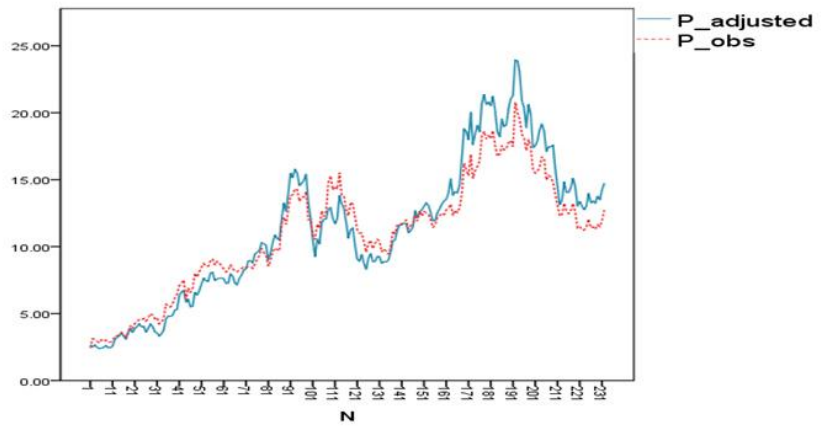

Fig. 4 The comparison of the adjusted model data and the observed data (SIC)
Table 4: Three indicators of SIC Pricing afer adjusting

\begin{tabular}{|c|c|c|c|}
\hline & MAE & MRPE & MAPE \\
\hline Mean & 0 & 0 & .0563 \\
\hline $\mathrm{N}$ & 232 & 232 & 232 \\
\hline Standard Deviation & 1.01266 & .14471 & .09187 \\
\hline
\end{tabular}

\section{CONCLUSIONS}

In this paper, the GARCH $(1,1)$ model is used for data modeling and forecasting. The $\operatorname{GARCH}(1,1)$ model is combined with the Mote Carlo algorithm to study European option pricing. The differences between the model data and the observed data are calculated by the GARCH-MC model. The GARCHMC model is adjusted according to the differences to forecast the option prices. The GARCH-MC model is tested by the SGCOC and the SIC warrants. The conclusions are following:

(1) The variation trend of the model data and the observed data of the SGCOC and the SIC warrants are coincident.

(2) In the SGCOC pricing, the differences between the model data and the observed data are large. It shows the GARCH-MC model can't be used directly to forecast its prices. But after adjusting, the GARCH-MC model is fit for the SGCOC option pricing.

(3) In the SIC pricing, the differences between the model data and the observed data are small. It shows the GARCH-MC model can be used directly to forecast its prices. But after adjusting, the model accuracy is higher.

\section{REFERENCES}

[1] Engle \& Robert F., Autoregressive Conditional Heteroskedasticity with Estimate of the Variance of U.K. Inflation. Ecomometrica, (50), pp.987-1008, 1982.

[2] Bollersleve \& Tim, Generalized Autoregressive Conditional Heteroskedasticity. Journal of Econometrics, (31), pp.307-327, 1986.

[3] Taufiq Choudhry, Integrate-GARCH and Non-stationary Variances: Evidence from European Stock Markets during 1920s and 1930s.Economics Letters, (1), pp.55-29, 1995.

[4] Cong Li, VAR Analysis under Different Distribution Study Based on GARH Model. Statistics and Decision, (9), pp.79-80, 2006.

[5] Jui-chung Hung, A Fuzzy Asymmetric-GARCH Model Applied to Stock Markets Information Sciences, (22), pp.3930-3934, 2009.

[6] Hafner. C.M \& J.V.KRombouts, Semiparametric multivariate volatility models. Econometric Theory, (26), pp.77-81, 2007.

[7] Lanne.M \& P Saikkonen, A multivariate generalized orthogonal factor GARCH model. Journal of Business, (33), pp.122-124, 2007. 